\title{
Carotenoid metabolism in animals: a biochemist's view
}

\author{
Brian H. Davies \\ Department of Biochemistry and Agricultural Biochemistry, The University College \\ of Wales, Penglais, Aberystwyth, Dyfed, SY23 3DD, U.K.
}

\begin{abstract}
Carotenoid metabolism in animals takes place as a result of enzymes which catalyse three main types of reaction. These main reaction types are (i) the substitution of carotenoid end groups (often $\beta$-end groups) by oxygen functions $(-\mathrm{OH}$ and $-\mathrm{C}=\mathrm{O})$, (ii) the alteration of end groups, e.g. of $\beta$ to $\varepsilon$, of $\beta$ to $k$, etc. and (iii) cleavage of the polyene chain to yield apocarotenoids and even the vitamins $A$. Perhaps all three main reaction types are represented in the formation of the oil droplet carotenoids in the retinas of birds.
\end{abstract}

\section{INTRODUCTION}

It does not seem either appropriate or useful, in a year which has seen the publication of an important new monograph on the biochemistry of animal carotenoids (Ref. 1) to attempt yet another overall review of this increasingly-active area of carotenoid biochemistry Rather, I would like to highlight some of those questions relating to the metabolism of carotenoids in animals which are posed for the biochemist by the natural product organic chemist on the one hand, and by biologists on the other. The organic chemist's questions were enumerated by Buchecker (2) at the last symposium in this series. It has to be said that the intervening three years have seen but few new answers emerging from biochemistry laboratories. This is because the biochemist, by the very nature of the discipline, is committed to a longer term preparation for his/her work which will, eventually, answer such questions. The questions raised in that one review (Ref. 2) alone will probably take at least a decade to be answered properly in terms of biochemistry.

Advancements in physico-organic techniques now enable organic chemists to describe newlyisolated carotenoids in terms of remarkable new dimensions of structural detail. In particular, chiroptical techniques can define the structures in terms of absolute stereochemistry. Against this background, it seems a pity that papers are still appearing in the carotenoid literature in which carotenoids are "identified" merely by an "identical absorption spectrum" or "consistent chromatographic behaviour".

Thus there are areas of the animal carotenoid literature which are in something of a mess; these areas have to be recognized and approached, entered, and then explored carefully because there is a "jungle" of "mis-identifications" therein. The inexperienced explorer should be wary and should take the trouble to be equipped with (or at least, obtain access to) the best physico-organic equipment available. Such adventures nowadays require the support of a sound "back-up" group, namely the appropriately-experienced organic chemists who use such equipment routinely. I have always found them very willing to collaborate with a biologist in ensuring that structures are correct in every detail.

The role of the biochemist, in one sense, is to prove that a putative arrow, joining two adjacent organic structures in a speculative sequence, really represents an actual metabolic reaction in the living organism concerned. But, also, the biochemist has to prove these reactions in the best possible way, by using enzyme systems as well as whole-animal feeding trials, and by using appropriate means of labelling the actual carotenoid molecules added to the feed or used as enzyme substrate. Plant and microbial carotenoid biochemistry (i.e. carotenoid biosynthesis) are both much better understood as a result of the preparation and use of cell-free enzyme systems. Our understanding of animal carotenoid biochemistry (i.e. metabolism) will only catch up when enzyme systems from animal tissues are used as experimental tools. To do this should be the goal of any animal carotenoid biochemist. With a single exception, the enzymes of animal carotenoid metabolism are unknown, and little investigated. Yet their products, animal carotenoids of known structure, increase in number, variety and complexity all the time (see other contributions to this volume). 
THE SOURCE OF ALL ANIMAL CAROTENOIDS: THE PLANT KINGDOM

The assumption is made that animals cannot form their own carotenoids de novo and that the carbon skeletons of all animal carotenoids are ultimately of plant origin, being derived via the "food chain". While the assumption is well-founded in the literature (Refs. 1 , 2 , 3), the precise inability of animals with regard to carotenoid biosynthesis has not been defined clearly. Animals are perfectly capable of forming geranylgeranyl pyrophosphate (GGPP), and, given the low substrate specificity of squalene synthase (Ref. 4) which normally catalyses the reductive dimerization of farnesyl pyrophosphate to squalene, ought to be metabolically capable of forming lycopersene $\left(7,8,11,12,15,7^{\prime}, 8^{\prime}, 11^{\prime}, 12^{\prime}, 15^{\prime}\right.$-decahydro- $\psi, \psi$-carotene. Presumably it is the oxidative dimerization of GGPP to phytoene $\left(7,8,11,12,7^{\prime}, 8^{\prime}, 11^{\prime}, 12^{\prime}\right.$-octahydro- $\left.\psi, \psi-c a r o t e n e\right)$ which they cannot manage. There are certainly reports (see e.g. Ref. l) of phytoene and other known intermediates in carotenoid biosynthesis being found in animals; it is always assumed that these are obtained per se from dietary plant materials.

This dietary dependence of animals upon plants is all the more remarkable when one considers the importance in the animal kingdom of the sense of vision. The visual process has one fundamental photochemical reaction, the llz/llE isomerization of retinal. The sole source of retinal to the animal is by the cleavage of carotenoid molecules obtained via the diet from plants.

Thus cleavage of the polyene chain of dietary carotenoids is one way in which animals metabolise carotenoids. The other ways are by substituting oxygen functions, usually in B-end groups, and by modifying the nature of the end groups. I propose to discuss these three types of reaction separately, and then to show how all are involved in considering another aspect of animal vision, the carotenoid-containing oil droplets of the avian retina.

\section{SUBSTITUTION OF THE $\beta$-END GROUP BY OXYGEN FUNCTIONS}

It is always important to look back at old experiments, in the hope that knowledge more recently gained will help to give a better interpretation of the old results. One of the commonest reactions characteristic of (but not exclusive to) carotenoid metabolism in animals is the substitution of the $\beta$-end group by a carbonyl oxygen at C-4. This is seen at its simplest in those (Californian) strains of the brine shrimp, Artemia salina, in which canthaxanthin ( $\beta, \beta$-carotene-4,4'-dione) is the most highly oxygenated carotenoid. While canthaxanthin itself may well be a natural component of this crustacean's diet, an investigation was initiated 20 years ago into whether Artemia is capable of converting $\beta$-carotene into canthaxanthin (Ref. 5). A method was found of rearing Artemia nauplii on a carotenoid-free diet (of bakers' yeast) so that endogenous carotenoid levels were low after three weeks of this dietary regime. The feeding of unlabelled $\beta$-carotene over six days led to the formation of both echinenone ( $\beta, \beta$-caroten-4-one) and canthaxanthin. A further six days on the carotenoid-free diet led to canthaxanthin becoming the major carotenoid of the nauplii. Thus, only after 12 days was the quantitative conversion of $\beta$-carotene into canthaxanthin demonstrable; if the organism itself takes so long to metabolize the dietary carotenoid, the chances are slim of doing a proper biochemical experiment using an enzyme system in which cofactor requirements could be demonstrated in order to point to a possible reaction mechanism.

The Artemia experiment was repeated, this time using [ $\left.{ }^{14} \mathrm{C}\right]$ - $\beta$-carotene prepared, using greening etiolated maize seedlings, from ${ }^{14} \mathrm{CO}_{2}$ ( $\operatorname{Ref} .6$ ). Good incorporations were obtained into both echinenone and canthaxanthin. That good incorporations were demonstrable using weakly-labelled $\beta$-carotene $(12.4 \mu \mathrm{Ci} / \mathrm{mg})$ was due to low endogenous carotenoid levels being established in the animals before feeding was commenced. We know from later work on prawns and lobsters (Ref. 7), and from some very recent work with goldfish (Ref. 8) how difficult it is to demonstrate carotenoid conversions in such animals with high endogenous carotenoid levels. The use of high specific-activity carotenoid substrates is essential.

A third feature of the early Artemia work deserves comment. Feeding trials were also made with unlabelled isocryptoxanthin $(\beta, \beta$-caroten-4-ol) and isozeaxanthin ( $\beta, \beta$-carotene$4,4^{\prime}$-diol). Both were absorbed; the isocryptoxanthin was converted into $4^{\prime}$-hydroxyechinenone (4'-hydroxy- $\beta, \beta$-caroten-4-one), but no further; the isozeaxanthin was not metabolized (Ref. 6). Nor was either of these compounds detected during the feeding trials with $\beta$-carotene. These observations were considered unusual at the time because isocryptoxanthin is the logical intermediate between $\beta$-carotene and echinenone. 
Work in other laboratories and with other crustaceans has been able to implicate isocryptoxanthin and isozeaxanthin, which have been detected in a number of situations (see Ref. I for details). Reports of the isolation of isocryptoxanthin or isozeaxanthin, however, now have to be interpreted with some caution since it has been shown that Micro-Cel C, a common chromatographic adsorbent in many carotenoid laboratories, can cause artifact formation (Ref. 9). $\beta$-Carotene, for instance, is converted almost quantitatively into 3,4-didehydro- $\beta$-carotene, isocryptoxanthin and isozeaxanthin on passing through Micro-Cel C (Ref. 10). A reaction scheme has been proposed (Ref. 11).

Yet there must be a hydroxylated intermediate in the formation of a ketone at c-4. Such reactions in other areas of biochemistry all take place in two stages, namely (a) the action of a mono-oxygenase to form the secondary alcohol and (b) the dehydrogenase-catalysed oxidation to the ketone. A proposal made to explain the Artemia results at the time (Ref. $6)$ is still valid. One end of the $\beta$-carotene molecule combines with an enzyme which could be a mono-oxygenase/dehydrogenase multiplex. All reactions take place while the carotenoid is attached to the protein and dissociation of the complex yields free enzyme and the 4-keto-carotenoid. Access to the enzyme is not possible other than by a $\beta$-end group unsubstituted at $\mathrm{C}-4$. Thus isocryptoxanthin and isozeaxanthin are not metabolized when fed, and are undetectable as intermediates because of their merely transient existence in the enzyme complex.

When the formation of astaxanthin (3,3--dihydroxy- $\beta, \beta$-carotene-4, $4^{\prime}$-dione) in animals is considered, the situation becomes much more complex; many schemes have been postulated for routes from $\beta$-carotene to astaxanthin which take account of the many possible intermediates found in insects, crustacea, fish and birds (see Refs. 1,2). The main question is whether the $\beta$-carotene is first hydroxylated to zeaxanthin ( $\beta, \beta$-carotene$3,3^{\prime}$-diol) which is then a substrate for the enzymes which introduce carbonyl oxygens at C-4 and $4^{\prime}$, or whether canthaxanthin is formed first and is then hydroxylated at C-3 and $3^{\prime}$. Perhaps this is another situation like that previously illustrated for the photosynthetic bacterium Rhodospipillum rubrum (Ref. 12) which has a whole network of different pathways all of which lead, but by different routes and using different intermediates, from the same precursor and to the same product. In astaxanthin formation too, only three enzymes, each of broad substrate specificity, need be involved, a C-3 mono-oxygenase, a C-4 mono-oxygenase and a caroten-4-ol dehydrogenase.

This situation can only be resolved when enzyme systems able to effect the required transformations have been prepared. Over the years we have sought enzyme systems capable of the formation of astaxanthin. Perhaps one such attempt deserves mention here. Peter Donkin, working in my laboratory over ten years ago, incubated [ $\left.{ }^{4} \mathrm{C}\right] \beta$-carotene with buffer extracts of lobster epidermis and hepatopancreas and those cofactors which we thought might be involved in the metabolism of $\beta$-carotene. We were unable to demonstrate any conversion of $\beta$-carotene into astaxanthin, but there was a low incorporation of label into both echinenone and canthaxanthin (Ref. 7).

That was a few years ago, before we were conscious of carotenoid chirality, which must arise in natural carotenoids from the stereospecificity of the enzymes concerned. Astaxanthin exhibits a number of peculiarities in this respect which are deserving of biochemical consideration. When astaxanthin occurs in the plant kingdom, it is stereochemically-pure, but not always of the same chirality. Thus the astaxanthin formed as a secondary carotenoid when the green alga Haematococcus pluvialis is put under stress is $>998\left(3 \underline{S}, 3^{\prime} \underline{S}\right)$ (Ref. 13), while the astaxanthin from the red yeast Phaffia rhodozyma is $>98 \%\left(3 \underline{R}, 3^{\prime} \underline{R}\right)$ (Ref. 14). That the two astaxanthins are of opposite chiralities has to reflect the stereospecificity of the enzymes responsible for the 3-hydroxylations; perhaps the direction of attack is influenced by whether or not the $\beta$-end group is already carrying a carbonyl oxygen at C-4 (Ref. 15).

Even more deserving of a rational biochemical explanation is the stereochemistry at C-3 and $3^{\prime}$ of the astaxanthin from a number of animal species (lobster, shrimp, salmon) where the astaxanthin has been shown to be a mixture of $\left(3 \underline{R}, 3^{\prime} \underline{R}\right),\left(3 \underline{S}, 3^{\prime} \underline{S}\right)$ and the meso-form $\left(3 \underline{R}, 3^{\prime} \underline{S}\right)$. The proportions of the three types are not the same in the different species (Ref. 16). The question as to whether the astaxanthin molecules are formed in three different ways, or whether biosynthesized optically-pure, the astaxanthin is then subject to action by isomerizing enzymes, is probably already settled. A following paper at this Symposium, by K. Schiedt, F.J. Leuenberger, M. Vecchi and E. Glinz, shows that the stereochemistry of the enantiomeric forms of dietary astaxanthin is retained on absorption by the tissues of the rainbow trout, so astaxanthin cannot be the substrate of an active C-3/3' epimerase. Thus stereochemistry must reflect biosynthesis alone. If the direction of the 3- (or $3^{\prime}-$ ) hydroxylation of the $\beta$-end group is influenced by whether or not the ring has a 4-oxo-substituent, and if the formation of astaxanthin from $\beta$-carotene is the result of the three enzymes described above working in an undefined order, with no single reaction sequence operating, then the result would be a mixture of the two enantiomeric astaxanthins and meso-astaxanthin. The ratio of the three forms would be dependent on the relative activities of the two mono-oxygenases prevailing in the organism concerned. 
The same sort of question has arisen in the case of those insect carotenoids which have a 2-hydroxy-substitution of the $\beta$-end group. The carotenoids in question are $\beta, \beta-c a r o t e n-$ 2-ol of the moth Cemra vinula and $\beta, \beta-\operatorname{caroten}-2-o l, \beta, \beta$-carotene-2,2'-diol, and 2'-hydroxy$\beta, \beta$-caroten-2-one in the stick insect, Ectatosoma tiaratum. All are partial racemates (Refs. 17, 18). The formation of the carotene-2-ol, the 2,2'diol and the 2'-hydroxy2-ketone occurs during development of the Ectatosoma embryo, and mirrors a decline in the levels of $\beta, \beta$-caroten-2-one and $\beta, \beta$-carotene-2,2'-dione (Ref. 17). These kinetics imply a precursor/product relationship for the 2-one and 2-ol which is unusual, oxygen normally being introduced in Nature as a hydroxyl group which is then oxidised (dehydrogenase). Two possible explanations have been suggested for the racemic nature of the 2-ols. In one, they are formed from the 2-ones by a non-stereospecific dehydrogenase, while the second suggests that hydroxylation occurs first to give an optically-pure 2-ol which is then epimerized via the 2-one, presumably by two stereospecifically-different stereospecific dehydrogenases. The second explanation, avoiding the concepts of direct carbonyl introduction into the $\beta$-end group, and of a nonstereospecific enzyme, would seem biochemically the more acceptable.

\section{CONVERSIONS OF CAROTENOID END GROUPS}

The distribution of the $k$-end group among plant carotenoids is limited, and the occurrence of such carotenoids is confined to but a few species (Ref. 19). Typical carotenoids are those of the fruits of the red pepper, Capsicum annuum, namely capsanthin (3,3'-dihydroxy$B, K-$ caroten $-6^{\prime}-$ one $)$ and capsorubin $\left(3,3^{\prime}\right.$-dihydroxy- $k, k$-carotene- $\left.6,6^{\prime}-d i o n e\right)$. The $k-e n d$

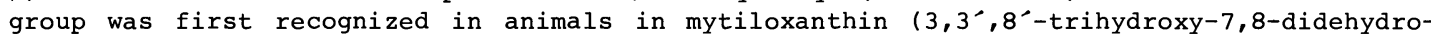
$\beta, K$-caroten $-6^{\prime} 8^{\prime}$-dione), a characteristic carotenoid of the common mussel, in which the K-end group is associated with a 6,8-diketone (Ref. 20). This same combination occurs in sponge carotenoids such agelaxanthin C (7,8-didehydro-19,3', $8^{\prime}$-trihydroxy-3 [?]-methoxy$\beta, k$-caroten-6'-one), trikentriorhodin (3-hydroxy-7,8-dihydro-k, x-carotene-6,8-dione) and trikentriophidin (3-hydroxy-7,8-dihydro-k, $\phi$-carotene-6,8-dione) (Ref. 21). Other sponge carotenoids, the bastaxanthins, contain $k$-end groups with just a 6-ketone (Ref. 22).

It is suggested that the 6-oxo-k-end groups of capsanthin and capsorubin are formed by pinacollic rearrangements of 5,6-epoxy- $\beta$-end groups (Ref. 23), and there is some evidence to support this from mutant studies in plants (Ref. 24). Presumably the same type of rearrangement would form the 6-oxo-k-end groups of the bastaxanthins. Fucoxanthin (5,6-

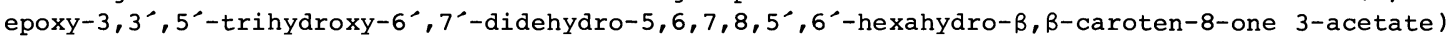
a carotenoid of the marine diatoms comprising part of the diet of the mussel, already has an 8-oxo substitution and could well be the precursor of mytiloxanthin in the mussel (Ref. 2). Experimental proof of this is currently being sought at Aberystwyth as part of a project funded by the Natural Environment Research Council.

An early part of that project is now complete. This was a reinvestigation of the alleged effect of low oxygen tension (e.g. in polluted waters) in increasing the apparent carotenoid content of Mytilus galloprovincialis, the carotenoid colour doubling in a mere $10-15 \mathrm{~h}$ (Ref. 25). This was interpreted in terms of the carotenoids having a function as oxygen carriers, taking up oxygen when available (e.g. at high water) to form colourless in-chain epoxides or dioxides, and then releasing the oxygen under anoxic conditions (e.g. at low water), with a concomitant restoration of the polyene chain chromophore, in order to make the oxygen available for ATP production. Peter Raines, working at Aberystwyth has not been able to substantiate this with Mytilus edulis (Ref. 26), so the function of the carotenoids of our local mussels is still unknown.

Returning briefly to the sponge carotenoids, an explanation is still sought for the formation of the aromatic rings. One view is that 3,4-didehydrogenated $\beta$-end groups are involved; this is supported by the presence of carotenoids with appropriate structures (Ref. 21).

Actinioerythrin $\quad\left(3,3^{\prime}\right.$-dihydroxy-2,2 - dinor- $\beta, \beta$-carotene-4, $4^{\prime}$-dione $3,3^{\prime}$-diacylate $) \quad$ is the most significant representative of a class of carotenoids characteristic of sea anemones, the 2-nor-carotenoids. Actinioerythrin can be visualised as arising from the benzylic rearrangement of a hypothetical triketone formed from astaxanthin. This has been discussed in some detail previously (Ref. 2). 2-Norastaxanthin, a possible intermediate, has been found in two Actinia spp. (Refs. 27, 28), but no work of a biochemical nature is reported.

Although the $\varepsilon$ - and $\beta$-end groups of carotenoids in the plant kingdom are metabolically immutable once they are formed, the same cannot be said of them in the context of animal carotenoids. The very existence of such $3,3^{-}$-dihydroxy- $\varepsilon, \varepsilon-c a r o t e n e s$ as the (6R) chiriquixanthins $A$ and $B$ in amphibia, and the (6ㅁ) tunaxanthins in fish, and the absence of such compounds from the respective diets, implies that the $\varepsilon-r i n g s$ are formed by animals, presumably from $\beta$-rings. The many stereochemical implications of such conversions have 
been discussed (Refs. 1, 2) but the reactions yet have to be proved. There is good evidence from the Californian sheephead fish (Pimelometopon pulchrum), which undergoes a spectacular colour change from golden-yellow to bright red (Ref. 3), that tunaxanthin is converted, probably via zeaxanthin, into astaxanthin (Ref. 29); a new study of this phenomenon using chiroptical methods would provide a wealth of new information.

There is evidence, too, for the formation of $\varepsilon$-rings in birds. The presence among the retinal oil droplet carotenoids of $\left(6 \underline{S}, 6^{\prime} \underline{s}\right)-\varepsilon, \varepsilon$-carotene as the sole carotene (Ref. 30) is the strongest circumstantial evidence for this $(\varepsilon, \varepsilon$-carotene is also the sole carotene of turtle oil droplets, but its chirality is undefined; Ref. 31). Direct evidence of $\varepsilon$-ring formation in birds comes from the demonstration that labelled zeaxanthin fed to laying hens undergoes conversion to $\left(6 \underline{S}, 6^{\prime} \underline{s}\right)-\varepsilon, \varepsilon-$ carotene- $3,3^{\prime}$ dione and the intermediate ( $\left.3 \underline{R}, 3^{\prime} \underline{s}\right)-3$-hydroxy- $\beta, \varepsilon$-carotene- $3^{\prime}$-one, both of which appear in the egg yolks (Ref. 32).

\section{CLEAVAGE OF CAROTENOIDS}

That animals are capable of cleaving the polyene chain of carotenoids is clear from the presence in a few species of some of the lower animal orders of such carotenoids as hopkinsiaxanthin (3-hydroxy-7,8-didehydro-7 $7^{\prime}, 8^{\prime}$-dihydro-7'-apo- $\beta$-carotene-4, $8^{\prime}$-dione ; Ref. 33) and paracentrone (3,6-dihydroxy-6,7-didehydro-5,6, $7^{\prime}, 8^{\prime}$-tetrahydro- $7^{\prime}$-apo- $\beta$ caroten-8-one; Ref. 34). The galloxanthin from avian retinas has now been confirmed as (3R) $-10^{\prime}$-apo- $\beta$-carotene-3,10'-diol after the synthesis of that structure in Pfander's laboratory.

There is only one enzyme concerned with the metabolism of carotenoids in animals which has been studied thoroughly; this is the mammalian intestinal $\beta$-carotene 15,15'-dioxygenase which cleaves $\beta$-carotene to yield two molecules of retinal. The suggestion that the enzyme should, more properly, be called carotenoid 15,15'-dioxygenase, stems from its wide substrate specificity (Ref. 35). Its mechanism probably involves the addition of a molecule of oxygen across the central $15,15^{\prime}$-double bond (of $\beta$-carotene); this is supported by labelling studies (Refs. 36, 37).

The metabolism of carotenoids to yield 3,4-dehydroretinol (vitamin $A_{2}$ ) in freshwater fish is of current interest. Earlier studies suggested that xanthophylls based on $\beta$-carotene are precursors (Ref. 38) while a more recent view is that lutein $\left(\beta, \varepsilon-\right.$ carotene- $3,3^{\prime}-$ diol $^{\prime}$ ) is the true precursor (Ref. 39). The accompanying contribution by Mrs $K$. Schiedt and her collaborators shows quite clearly that labelled vitamins $A_{1}$ and $A_{2}$ are formed in trout from any of a number of labelled carotenoids, zeaxanthin, canthaxanthin and astaxanthin. Bethan Davies, working in my laboratory has shown recently that goldfish, too, incorporate radioactivity from labelled canthaxanthin into retinol and dehydroretinol. Thus earlier observations (Ref. 38) are confirmed; it is clear that fish, exceptionally, are able to metabolise carotenoids reductively as well as oxidatively.

\section{CAROTENOIDS OF AVIAN RETINAL OIL DROPLETS}

Identifications of the carotenoids of the oil droplets of avian retinas have been reported for the turkey (Ref. 30); the carotenoids of the retinas of chickens, ducks and geese are qualitatively the same (Ref. 40). One of the carotenoids originally described as "14'-apo- $\beta$-caroten-14"-al" (Ref. 41) turned out not to have that structure and was an artifact anyway (Ref. 42). The carotenoids present in turkey retinas are lutein, zeaxanthin, astaxanthin, galloxanthin, $\left(6 \underline{S}, 6^{\prime} \underline{S}\right)-\varepsilon, \varepsilon$-carotene and a $\varepsilon, \varepsilon$-carotene-3, $3^{\prime}-$ diol.

The presence of lutein and zeaxanthin is unremarkable; both are constituent carotenoids of many avian tissues. The presence of astaxanthin (chirality unknown), galloxanthin and $\left(6 \underline{S}, \sigma^{\prime} \underline{S}\right)-\varepsilon, \varepsilon$-carotene, however, pose some questions about their metabolic origin. As the variously-coloured droplets are present in the retina of the newly-hatched chick, it is clear that the three novel carotenoids originate from carotenoids in the egg yolk, i.e. zeaxanthin and lutein.

(3R, $3^{\prime} R$ )-Zeaxanthin can be visualised as the precursor. It could be converted into astaxanthin by oxidations to carbonyls of $\mathrm{C}-4$ and $4^{\prime}$, and its conversion into galloxanthin by chain cleavage would be consistent stereochemically. The formation of carotenoids with (6S)- $-\varepsilon$-rings from zeaxanthin by the chicken is established (see above; Ref. 32 ). Studies to test these three hypotheses are well under way. A method has been devised by Alan Akers and Bethan Davies for introducing labelled zeaxanthin into the yolk of fertile eggs, and the mobilization of radioactivity into the chick embryo retinal lipid has been shown to coincide precisely with the formation of the differently-coloured oil droplets, on the 19th day of incubation (Ref. 43). 
ACKNOWLEDGEMENTS

I would like to thank those students and collaborators, past and present, aspects of whose work have been included in the above account. They are Alan Akers, Bethan W. Davies, Peter Donkin, Rosemary J. Lewis-Jones (nee DeB. Apps), Susan Pollard, Peter S. Raines and Ann J. Shufflebotham. Some of the studies described were supported by grants from the Science and Engineering Research Council and the Natural Environment Research Council. Help from Dr. H. Pfander (University of Berne) and from Dr. K. Bernhard and Mrs. K. Schiedt and their colleagues at F. Hoffmann-La Roche \& Co. Ltd., Basle, is much appreciated.

\section{REFERENCES}

T.w. Goodwin, The Biochemistry of the Carotenoids, 2nd. Edn., Vol. 2 (Animals), Chapman \& Hall, London (1984).

R. Buchecker, in Carotenoid Chemistry and Biochemistry (G. Britton \& T.W. Goodwin, eds.), pp. 175-193, Pergamon, Oxford (1982).

D.L. Fox, Animal Biochromes and Stmuctural Colours, 2nd Edn., University of California Press, Berkeley (1976).

A.A. Qureshi, F.J. Barnes, E.J. Semmler and J.W. Porter, J. Biol. Chem. 248, 2755-2767 (1973)

W-J. Hsu, C.O. Chichester and B.H. Davies, Comp. Biochem. Physiol. 32, 69-79 (1970).

B.H. Davies, W-J. Hsu and C.O. Chichester, Comp. Biochem. Physiol. 33, 601615 (1970).

P. Donkin, Ph.D. Thesis, University of Wales (1978).

B.H. Davies and B.W. Davies: unpublished work.

D.B. Rodriguez, Y. Tanaka, T. Katayama, K.L. Simpson, T-C. Lee and C.O. Chichester, J. Agric. Food Chem. 24, 819-822 (1976).

R.P. Ritacco, D.B. Rodriguez, G. Britton, T-C. Lee. C.O. Chichester and K.L. Simpson, J. Agric. Food Chem. 32, 296-300 (1984).

R.P. Ritacco, G. Britton and K.L. Simpson, J. Agric. Food Chem. 32, 301-304 (1984).

B.H. Davies, in Farbstoffe in Pflanzen (F-C. Czygan, ed.), pp. 7-25, Gustav Fischer, Stuttgart (1975).

B. Renstr $\phi$ m, G. Borch and S. Liaaen-Jensen, Phytochemistry 20, 2561-2564 (1981).

R.K. Müller, K. Bernhard, H. Mayer, A. Rüttimann and M. vecchi, Helv. Chim. Acta 63, 1654-1664 (1980).

A.G. Andrewes and M.P. Starr, Phytochemistry 15, 1009-1011 (1976).

R.K. Müller, K. Bernhard and M. Vecchi, in Carotenoid Chemistry and Biochemistry (G. Britton and T.W Goodwin, Eds.), pp. 27-54, Pergamon, Oxford (1982).

H. Kayser, in Carotenoid Chemistry and Biochemistry (G. Britton and T.W. Goodwin, eds.), pp. 195-210, Pergamon, Oxford (1982).

H. Kayser, K. Aareskjold, G. Borch and S. Liaaen-Jensen, Insect Biochem. 14, 51-54 (1984).

A. Rüttimann, in Carotenoid Chemistry and Biochemistry (G. Britton and T.W Goodwin, eds.), pp. 71-86, Pergamon, Oxford (1982).

A. Khare, G.P. Moss and B.C.L. Weedon, Tetrahedron Letters 40, 3921-3924 (1983).

M. Yamaguchi, in Carotenoid Chemistry and Biochemistry (G. Britton and T.W. Goodwin, eds.), pp. 225-235 (1982).

S. Hertzberg, T. Ramdahl, J.E. Johansen and S. Liaaen-Jensen, Acta Chem. Scand. B37, 267-277 (1983).

B.C.L. Weedon, in Carotenoids (0. Isler, ed), pp. 25-59, Birkhäuser, Basel (1971). B.H. Davies, S. Matthews and J.T.O. Kirk, Phytochemistry 9, 797-805 (1970).

V.N. Karnaukhov, Symp. Biol. Hung. 19, 151-167 (1979).

P.S. Raines and B.H. Davies, Abstr. 7th Int. Symp. Carotenoids, Munich (1984).

G.W. Francis, S. Hertzberg, R.R. Upadhyay and S. Liaaen-Jensen, Acta Chem. Scand. 26, 1097-1104 (1972).

J.D. Tauber, A. Fiksdahl and S. Liaaen-Jensen, Biochem. Syst. Ecol. 8, 437439 (1980).

G.F. Crozier, J. Exp. Mar. Biol. Ecol. 4, 1-8 (1969).

B.H. Davies, Pure Appl. Chem. 51, 623-630 (1979).

R.J. deB. Apps, A. Akers, and B.H. Davies, Abstr. 6th Int. Symp. Carotenoids, Liverpool (1981).

K. Schiedt, G. Englert, K. Noack, M. Vecchi, and F.J. Leuenberger, Abstr. 6th Int. Symp. Carotenoids, Liverpool (1981).

J.w. McBeth, Comp. Biochem. Physiol. 41B, 55-68, 69-77 (1972).

G. Galasko, J. Hora, T.P. Toube, B.C.L. Weedon, D. André, M. Barbier, E. Lederer and V.R. Villanueva, J. Chem. Soc. C., 1264-1265 (1969).

J.A. Olson, in Biosynthesis of Isoprenoid Compounds (J.W. Porter and S.L. Spurgeon, eds.), Vol. 2, pp. 371-412, John Wiley, New York (1983).

D.S. Goodman, H.S. Huang and T. Shitatori, J. Bioi. Chem. 241, 1929-1932 (1966).

B.B. Vartapetyan, A.A. Dmitrovskii, D.G. Alkhazov, I.K. Lemberg, A.B. Girshin, G.M. Suisinskii, N.A. Starikova, N.N. Erofeeva and I.P. Bogdanova, Biokhimiya 31, 881-886 (1966).

J. Gross and P. Budowski, Biochem. J. 101, 747-754 (1966).

A.B. Barua and U.C. Goswami, Biochem. J. 166, 133-136 (1977).

S. Pollard, A. Akers, R.J. Lewis-Jones and B.H. Davies: unpublished work.

S. Pollard, Ph.D. Thesis, University of Wales (1980).

B.H. Davies and A.J. Shufflebotham: unpublished work.

B.W. Davies, A. Akers and B.H. Davies, Abstr. 7th Int. Symp. Carotenoids, Munich (1984). 